\title{
Mental image and mind's eye transformations of cutaneous drawings
}

\author{
REED W. MANKIN and ROBERT J. WEBER \\ Oklahoma State University, Stillwater, Oklahoma 74078
}

\begin{abstract}
In Experiment 1, mental orientation was studied by having subjects identify finger drawings of ambiguous letters (p, $q, b, d)$ on six areas of the head. Subjects identified the drawings from their own most natural orientation. Responses were scored as either "internal" (from an origin of orientation at the center of the head) or "external" (from an origin of orientation outside the head). Results suggested that at least two principal axes of orientation exist, back to front and top down. In Experiment 2, two strategies for operating on cutaneous images were studied. These were rotation of mental images and mind's eye transformation of orientation. Subjects identified ambiguous letters after performing one of four mental operations. Results showed the image transformation strategy to be both faster and more accurate than the mind's eye transformation of orientation. Possible relations between image transformations and allocation of attention resources are discussed.
\end{abstract}

There are two broad geometric strategies people may use when asked to change the way they view a visual image. First, hold the mind's eye in one position and rotate the image about some axis: $x, y$, or $z$. Second, transform the location of the mind's eye and hold the image "fixed" in one place. Although a result of each of these strategies may be functionally identical, the processes used seem quite different.

The concept of a "mind's eye" is best understood as a metaphor for the interpretive processes that extract information from the image buffer (Kosslyn, 1980). These interpretive processes behave as if they are a disembodied eye that can (1) move around the mental representation of the environment or (2) produce or interpret operations on the image representation.

Cutaneous drawings may be used to study these phenomena. If a figure, like the letter " $p$," is traced on the forehead of a subject, it can be perceived either as a "p" or as a "q," depending on whether the subject experiences the figure from an internal point of view or from an external point of view. In other words, based on the subject's response, it is possible to infer the orientation as internal or external. Orientation problems of this nature have some real-world analogues. Auto mechanics who hold images of automobile parts in their minds must be able to change orientations to their images depending on whether they are working above, beneath, or on a side of a car. Differing positions of realworld objects necessitate cognitive reorientation to images held in memory. These orientation processes may be

Send reprint requests to Robert J. Weber, Department of Psychology, Oklahoma State University, Stillwater, Oklahoma 74078. This research was supported in part by a grant from the Educational Research I'oundation, Oklahoma State University, to the second author. different from the mental rotation work of Cooper and Shepard (1973) in an important way: With mental rotation, there is the strong impression that the body is fixed and the object rotates. In the present work, there is often the impression that the object or form is fixed, whereas the body changes its point of view or orientation.

\section{EXPERIMENT 1}

Natsoulas and Dubanoski (1964) proposed a conceptual scheme to explain the orientation of perceiver to figures drawn on the subject's skin. The lowercase letters $p, q, b$, and $d$ were traced on the forehead, back of the head, and sides of the head. Consistent internal responses were found on the forehead. Consistent external responses were found on the back of the head. Both the left and right sides of the head revealed a manner of responding that was not dominated by either perspective. These results, together with results obtained when the head is turned $90 \mathrm{deg}$ left and right, were interpreted by a moving origin, "effort" hypothesis. That is, if the mind's eye must turn more than 90 deg to view an image, it will translate its location to an external perspective. Duke (1966) posited a frontal-plane hypothesis to explain similar results. Duke's hypothesis stated that symbols drawn upon the anterior or posterior surfaces of the body are perceived as if projected out in front of the subject on a transparent two-dimensional surface.

Is there a way of distinguishing between the moving origin internal-external hypothesis and the frontal-plane hypothesis? Experimentally, we need to distinguish whether the location of the mind's eye remains fixed behind the head (frontal-plane hypothesis) or whether it shifts between inside and outside depending on the body surface on which the figures are drawn (a moving origin hypothesis). The present experiment tests these two 
hypotheses by tracing letters on six areas of the head: forehead, back of head, top of head, under chin, left side, and right side.

\section{Method}

Subjects. Fifteen women and five men undergraduates at Oklahoma State University served as subjects. They received extra credit for participation and were tested individually by the same male experimenter in the same experimental room.

Stimuli. Stimuli were the lowercase letters $p, q, b$, and d. Stimuli presented on the left and right sides of the head were drawn on the temples, just in front of the ear. Stimuli presented on the top of the head were drawn immediately anterior of the crown. All letters were drawn in an imaginary area about $6 \mathrm{~cm}$ tall and $3 \mathrm{~cm}$ wide. The "tail" of the letter was always drawn first, followed by the "loop"; this was done in one continuous motion without lifting the finger from the head. The letters were drawn slowly and firmly at a rate of about $1.5 \mathrm{sec} /$ letter.

Procedure. Instructions included an explanation of the stimuli and the procedure. Subjects were told that the cutaneous drawings could be considered from different points of view, but that they should view the drawings from their own most natural or comfortable point of view.

Subjects kept their eyes closed throughout the experiment. The experimenter then proceeded to draw the letters on the head surfaces with his forefinger. The order of head surfaces was randomly determined for each subject. A given head surface received each of the four letters in random order before the next surface received any. Responses were recorded by the experimenter from verbal report in which subjects said one of the four letter names. A series of postexperiment questions were asked of each subject to obtain subjective data that would help verify whether subjects were adhering to instructions and determine how subjects described strategies used in identifying cutaneous tracings.

\section{Results}

Responses for each subject for each head area were converted into a single score. This score indicated whether the subject responded from a predominately internal perspective, an external perspective, or a mixed perspective. Subjects were classified as having an internal origin if three or four of their four responses per head area were consistent with an internal origin of orientation. In contrast, subjects were classified as having an external origin if three or four of their responses per condition were consistent with an external orientation. All other subjects were classified as having a mixed origin of orientation and were not considered in the analyses. Thus for the analysis, the number of subjects in each of the conditions equals the number of internal subjects plus the number of external subjects. Once these numbers were tabulated for each condition, a two-tailed independent binomial test for significance was used with a chance probability of .5 for a single trial.

Reponses on the forehead and chin areas showed a significant number of subjects with an internal origin $(P=12 / 14, p<.05$, on the forehead $P=14 / 16, p<.05$, on the chin). The back of the head and the top of the head showed a consistent external origin different from chance $(P=17 / 17, p<.01$, on the back; $P=16 / 16$, $\mathrm{p}<.01$, on the top). Stimuli traced on the left side and right side of the head produced an inconsistent mixture of internal and external orientations (for internal scores: $P=7 / 11, p>.05$, on the left side; $P=4 / 12$, $\mathrm{p}>.05$, on the right side).

\section{Discussion}

Stimuli that are drawn on the top of the head show a very regular response pattern. The point of origin is external and from above the head, which is an orientation inconsistent with the frontal-plane hypothesis of a single viewpoint directly behind the head. The results for under the chin are similarly consistent from the internal perspective. The moving origin "effort" hypothesis proposed by Natsoulas and Dubanoski (1964) would predict that tracings under the chin would be perceived from a mixed perspective because the mind's eye must rotate at least 90 deg to view that skin area. Previous hypotheses in their present forms do not allow for consistent results for stimuli that are presented on head areas other than the forehead and back of the head. Either the mind's eye can shift from internal to external origins of orientation or the frontal-plane hypothesis must be modified to include at least two tracks of movement for the mind's eye: forward and back, and up and down. An alternative, albeit untested, hypothesis locates the mind's eye behind and above the head. For this perspective, the mind's eye could consistently interpret tracing on the top and bottom of the head.

The results of the postexperiment questionnaire indicated that subjects were visualizing images from internal and from external origins of orientation. Subjects also consistently reported that they were viewing images from their most natural orientations rather than from an orientation determined by the experimenter.

\section{EXPERIMENT 2}

Results from Experiment 1 raised an important question. If the mind's eye can assume various locations with respect to cutaneous tracings, then how does mind's eye translation around a fixed image compare with image rotation, as in the Cooper and Shepard $(1973,1975)$ experiments?

The purpose of Experiment 2 was to compare two functionally equivalent strategies for transforming visual images. Two image transformation groups used transformation of the visual image, as in the Cooper and Shepard experiments. Two mind's eye transformation groups used transformation of the location of the mind's eye, as in Experiment 1. Based on pilot data, it was hypothesized that the image transformation strategy would result in greater speed and accuracy. Further, from Experiment 1, the specific operations for both strategies that are closest to the "default" mental perspective should result in better performance. Two head surfaces were used, the forehead and the left side.

\section{Method}

Subjects. Twenty-four male and 24 female undergraduate psychology students at Oklahoma State University were randomly assigned to four experimental groups, with an equal number of males and females in each group. All subjects ranged in age from 18 to 24 years old and were native speakers of the English language. The subjects were given extra credit in their courses in return for participation. An additional five subjects 
were discarded because of an inability to correctly perform the task.

Stimuli. Stimuli were the same four lowercase letters used in Experiment 1 and were presented in an identical manner.

Procedure. The two image transformation groups (forehead group and left side of the head group) were asked to make rotations of the mental image of a cutaneous drawing while keeping the location of the mind's eye stationary. The two mind's eye transformation groups (forehead group and leftside group) were asked to make translations of the mind's eye while keeping the image of the letter stationary. Functionally equivalent operations were executed in each of the two transformation strategy conditions. The four operations for each group were numerically coded to insure that all groups received the same cuing stimuli. For the image transformation groups, the four numerical cuing codes $(1,2,3,4)$ were defined as (1) normal (mind's eye stationary and located inside the head looking out), (2) mirror image (image rotated in the depth plane so that the mirror image of the original figure is seen), (3) upside down (image rotated in the picture plane so that it is upside down), (4) mirror + upside down (image flipped in the depth plane so that it appears upside down and backward). For the mind's eye transformation groups, the four numerical cuing codes $(1,2,3,4)$ were defined as (1) up/inside (mind's eye inside the head looking out, with the mind's eye in an upright orientation), (2) up/outside (mind's eye outside the head looking back toward the head, with the mind's eye in an upright orientation), (3) down/inside (mind's eye inside looking out; it is in an upside down orientation), (4) down/outside (mind's eye outside looking in; it is in an upside down orientation). These coded operations produce exactly the same functional effect for both image transformation and mind's eye transformation groups. Thus "mirror image" and "up/outside" produce the same result.

Four letters (p, q, b, d) were traced for each of the four operations. Two complete replications of the 16 unique combinations of letter and operation were given to each subject. Each replication, containing uniquely randomized presentations of the 16 combinations, immediately followed each other. In summary, the design consisted of two transformation strategies (image, mind's eye), two head surfaces (forehead, left side) and two sexes as between-subjects variables. Within-subjects variables were the four coded operations (normal or up/inside, mirror image or up/outside, upside down or down/inside, and mirror + upside down or down/outside), four letters ( $p, q, b, d)$, and two replications.

Subjects were first instructed about the details of the experiment, including the identities and meanings of the operation code numbers, the strategy for transforming the image or the mind's eye, and other aspects of the procedure. A 1 -ft-square glass plate with a printed lowercase letter $p$ was used to explain how different rotations or movements of the mind's eye could result in the perception of other letters $(q, b, d)$. Subjects in the mind's eye condition were instructed by witnessing a human figure placed in different orientations to the glass plate. All subjects were cautioned to respond as quickly but as accurately as possible, with the emphasis upon maintaining clear accurate visual images of the stimuli. Two series of practice trials were given to each subject during the instructional period, one for memorizing the numerical cuing codes and the second to insure that people were thoroughly familiar with the task.

After receiving instructions and training, subjects closed their eyes. The male experimenter traced a letter on the appropriate head area. Concurrent with the end of the tracing, the experimenter verbally gave one of the numerical cues for the required operation and started the clock. Subjects were asked to verbally name the letter at exactly the same time as they stopped the clock.

At the end of the study, all subjects were asked a short series of questions that were directed to the same purpose as those in Experiment 1.

\section{Results}

An analysis of variance was conducted on reaction times for correct responses. The most important result is indicated by the significant interaction between the two transformation strategies and the four operations $[F(3,120)=11.77, p<.001]$. Figure 1 displays the means, standard deviations, and percentage of errors for this interaction.

The image strategy subjects responded in a gradually slower fashion across the four operations (normal mean $=1.082 \mathrm{sec}$, mirror image $=2.004$, upside down $=$ 2.739 , and mirror + upside down $=2.969$ ). For NewmanKeuls multiple-range tests, critical differences (CDs) for significance are reported. The Newman-Keuls test revealed that none of these four operations was significantly different from its adjacent operation. Overall, though, the gradually increasing function of mean reaction times was significant with differences exceeding the value $(\mathrm{CD} 4=1.255, \mathrm{p}<.05)$. In contrast, the mind's eye group responded in a dramatically slower fashion across the four equivalent operations (up/inside mean = $1.851 \mathrm{sec}$, up/outside $=3.908$, down/inside $=6.271$, and down/outside $=7.507$ ). The Newman-Keuls test between

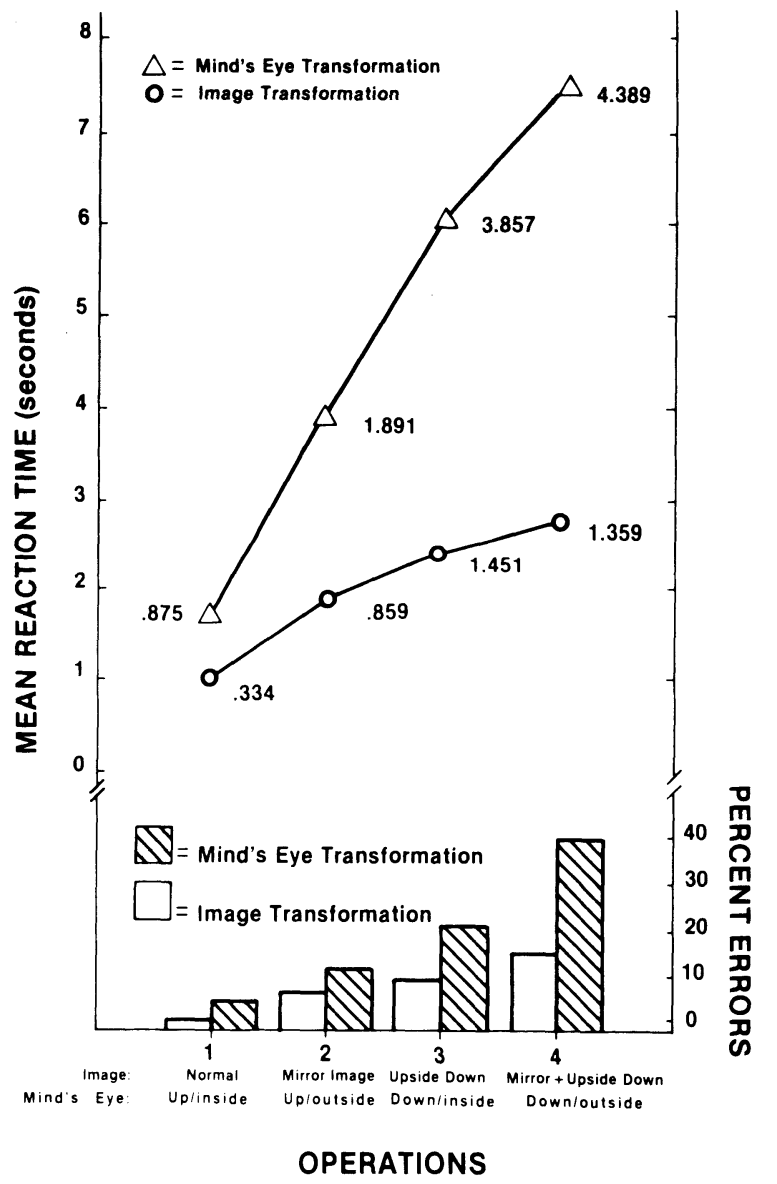

Figure 1. Experiment 2: Mean reaction times and error percentages for transformation strategies across operations. Standard deviations for time scores are adjacent to each point. 
these means revealed that each of the four operations was significantly slower than the preceding operation $(\mathrm{CD} 2=.955, \mathrm{p}<.05)$. The default operations for each strategy (normal for the image strategy and up/inside or up/outside for the mind's eye strategy) did not differ significantly from each other $(C D 2=1.219)$.

The percentages of errors for the Transformation by Operation interaction are given in the bottom right corner of Figure 1. For each mean, there were 192 responses. As shown, the range of errors was from $0 \%$ in the normal operation in the image transformation strategy to $39 \%$ in the down/outside operation in the mind's eye strategy. Error rates were positively correlated with reaction times over both transformations $(r=.95, p<.001)$. The patterns of errors showed that the majority of mistakes occurred when subjects responded by naming the mirror image of the correct letter. For the mind's eye transformation group, $84 \%$ of all errors were of this type. Among the image transformation subjects, this pattern of responding was $58 \%$. Analysis of reaction time data for errors indicated no speed-accuracy tradeoff. Overall, the reaction times for errors followed a pattern similar to that of correct responses, except that means for operations were approximately $25 \%$ higher.

Results between replications showed a significant practice effect $[F(1,40)=6.61, p<.05]$. Head area was not significant.

An important postexperiment question dealt with what the subjects "saw" in their imagination while trying to decide which letter was correct. Most of the image strategy subjects $(82 \%)$ reported that they clearly saw the letters rotating, flipping, or moving. Among the mind's eye strategy subjects, $87 \%$ claimed that they would mentally spin, rotate, or move around to achieve the correct perspective and then look at the letter from that perspective. Approximately $10 \%$ of the mind's eye subjects claimed that when they mentally stood on their heads, they would frequently lose sight of the letter. Others claimed that when they mentally moved around, the images of the letters would move or turn.

\section{Discussion}

The findings of the present study verified that image transformation with the mind's eye fixed in a stationary position is more efficient and effective than transformations in which the image is fixed and the mind's eye moves. These results support pilot data that suggested that people at times prefer to transform the image rather than the location of the mind's eye. This is not surprising when one considers the complexity of mind's eye transformations. Subjects' verbal reports suggest that translating the mind's eye requires at least two parallel processes: attending to the coordinate system through which the mind's eye is moving and attending to the target image. This hypothesis is supported by Jonides (1980), who documented a high cost of shifting attentional resources on images.

The interaction between transformation strategy and type of operation suggests that the mind's eye transformation is more sensitive to increased cognitive loads than is image transformation. As the mind's eye operations departed from the normal position, the speed and accuracy with which visual images could be manipulated deteriorated quite rapidly. Image transformation, on the other hand, remained relatively stable through various operations. It appears, then, that the structural processes associated with image transformations as opposed to mind's eye transformations are not disrupted as much by increased departure from default operations on mental images.

Finally, the proportion of errors made in naming letters from different operations increased with reaction times. This result is interpretable at least two ways. First, it appears that visual images require considerable maintenance (Kosslyn, 1980). The more complex operations in the study may require cognitive effort, so that routine refreshing of images is impaired. Second, concerning the high error rate for the mind's eye transformation, it is possible that incidental flipping or rotating of the letters occurred along with the translation of the mind's eye.

\section{GENERAL DISCUSSION}

The results of Experiment 1 indicate that there are clear default orientations for the different areas of the head. The results for Experiment 2 indicate that these default orientations can be overcome, but at some cost in time and accuracy. Several important questions arise. Are there features of particular images or of particular tasks involving images that govern whether an image strategy or a mind's eye strategy is employed? Under what conditions is each strategy most appropriate?

\section{REFERENCES}

Cooper, L. A., \& Shepard, R. N. Chronometric studies of the rotation of mental images. In W. G. Chase (Ed.), Visual information processing. New York: Academic Press, 1973.

Cooper, L. A., \& She PARd, R. N. Mental transformation in the identification of left and right hands. Journal of Experimental Psychology: Human Perception and Performance, 1975, 1, 48-56.

Duke, J. D. Perception of finger drawings upon the body surface. Journal of General Psychology, 1966, 75, 305-314.

Jonides, J. Towards a model of the mind's eye movement. Canadian Journal of Psychology, 1980, 34, 103-112.

Kosslyn, S. M. Image and mind. Harvard University Press: Cambridge, 1980.

Natsoulas, T., \& Dubanoski, R. A. Inferring the locus and orientation of the perceiver from responses to stimulation of the skin. American Journal of Psychology, 1964, 77, 281-285.

(Received for publication July 16, 1982.) 